\title{
Discord, quantum knowledge and private communications
}

\author{
Mile $\mathrm{Gu}^{1,2,3}$ and Stefano Pirandola ${ }^{4}$ \\ ${ }^{1}$ School of Physical and Mathematical Sciences, Nanyang Technological University, Singapore 639673 \\ ${ }^{2}$ Complexity Institute, Nanyang Technological University, Singapore 637723 \\ ${ }^{3}$ Centre for Quantum Technologies, National University of Singapore, 117543, Singapore \\ ${ }^{4}$ Computer Science $\&$ York Centre for Quantum Technologies, University of York, York YO10 5GH, UK
}

\begin{abstract}
In this brief review, we discuss the role that quantum correlations, as quantified by quantum discord, play in two interesting settings. The first one is discerning which unitaries have been applied on a quantum system, by taking advantage of knowledge regarding its initial configuration. Here discord captures the 'quantum' component of this knowledge, useful only when we have access to a quantum memory. In particular, discord can be used to detect whether an untrusted party has certain quantum capabilities. The second setting is quantum cryptography. Here discord represents an important resource for trusted-noise quantum key distribution and also provides a general upper bound for the optimal secret key rates that are achievable by ideal protocols. In particular, the (twoway assisted) secret key capacity of a lossy bosonic channel exactly coincides with the maximum discord that can be distributed between the remote parties at the two ends of the channel.
\end{abstract}

\section{KNOWLEDGE, CORRELATIONS, AND GUESSING CHANNELS}

The 1962 James Bond's movie 'Dr. No' taught children around the world a valuable lesson in how to detect whether nosy siblings are snooping into their rooms. You stick a small piece hair across the door and the doorframe. When the door is opens, the hair falls to the floor. The unsuspecting perpetrator has unwittingly communicated to you their rather unscrupulous action. This trick demonstrates the power of knowledge; by knowing how a system is initially configured (the location of hair), one can gain information about actions that have affected the system (opening the door).

This phenomena can be described by information theory. We denote a system of interest to be $A$, and knowledge about the system to be encoded within some memory $B$ - an approach previously adopted to understand uncertainty relations under quantum memory [1]. If $B$ contains information about $A$, the two systems will be correlated, such that $I(A, B)>0$.

The classical one time pad provides a simple example. Here Alice and Bob wish to communicate some secret message in the future. To do this, Alice and Bob gather in some secure location, where Alice generates a string of random bits that Bob commits them to memory. That is, they share many copies of the classically correlated state

$$
\rho=|00\rangle\langle 00|+| 11\rangle\langle 11| .
$$

Should Alice choose to flip some of her bits and give the resulting string to Bob, Bob is able to discern exactly which bits have been flipped by comparing the resulting string with the one stored in his memory. In contrast, anyone without access to Bob's memory would gain no information about Alice's actions. The optimal such scheme would allow Alice to communicate $\delta_{I}=1$ bit per copy of $\rho_{A B}$ shared. Thus possession of $B$ allows exclusive knowledge of how the system was manipulated. One notes that here, $I(A, B)=1$, which is equal to $\delta_{I}$. This is in fact, not a coincidence.

Consider the following general "channel guessing game".

1. Alice and Bob initially share a state $\rho$ distributed over the system of interest $A$, and the memory $B$. This initial state is publicly known.

2. Alice applies some unitary operator $U_{k}$ onto her subsystem $A$ with probability $p_{k}$. She publicly announces her protocol (e.g. the unitaries $U_{k}$ and their probability of application), but not the specific $k$ she selects in each run.

3. Alice gives $A$ to Bob, so that Bob is now in possession of $\rho_{A B}^{(k)}=U_{k} \rho_{A B} U_{k}^{\dagger}$. Without knowledge of $k$, Bob sees the ensemble state $\tilde{\rho}_{A B}=\sum_{k} p_{k} \rho_{A B}^{(k)}$.

4. Alice challenges Bob to guess which $U_{k}$ she has applied, i.e., to estimate the value of $k$.

This game captures a communication channel between Alice and Bob, where Alice has encoded a random variable $K$ that takes the value $k$ with probability $p_{k}$, onto corresponding codewords $\rho_{A B}^{(k)}$. The maximum information rate of this channel is then bounded above by the Holevo quantity

$$
I_{q}=\tilde{S}(A, B)-S(A, B),
$$

where $S(A, B)$ and $\tilde{S}(A, B)$ represent the respective entropies of $\rho_{A B}$ and $\tilde{\rho}_{A B}$. Here we consider the i.i.d. limit of many trials, where Alice repeats this game a large number of times; the performance of Bob, as quantified by the maximum information per trial, then saturates $I_{q}$.

This relation has a nice interpretation. In fact $\tilde{\rho}$ describes the state of the bipartite system after encoding, as viewed by an observer who is unaware of which $k$ was encoded in each run. Therefore $\tilde{S}(A, B)-S(A, B)$ captures the gain in entropy (or alternatively, the cost in 
negentropy) of encoding $K$ from their perspective. Thus Eq. (2) tells us that communication of $k$ bits of data necessarily incurs a minimum entropic cost of $k$.

Suppose Bob cannot access his memory (e.g. it was lost), the effective codewords would now be be $\rho_{A}^{(k)}$, with associated Holevo quantity

$$
I_{0}=\tilde{S}(A)-S(A) .
$$

The impact of having memory on Bob's in performance at the i.i.d. limit is then

$$
\Delta_{q} \equiv I_{q}-I_{0}=I(A, B)-\tilde{I}(A, B),
$$

where $I(A, B)$ and $\tilde{I}(A, B)$ are the respective mutual information of $\rho_{A B}$ and $\tilde{\rho}_{A B}$. The quantity $I(A, B)-$ $\tilde{I}(A, B)$ then represents the cost, in terms of total correlations between $A$ and $B$ of encoding $K$. Meanwhile $\Delta_{q}$ represents information about $K$ that is exclusively available to Bob due to his possession of $B$.

If we consider $I(A, B)$ to capture the amount of knowledge Bob knows about $A$, we find an interesting resource based view of knowledge: Bob can expend $k$ bits of knowledge about a system $A$ to learn at most $k$ bits of information about actions on $A$; in the i.i.d limit, this bound can be saturated. That is, knowing $k$ bits about some system $A$, as captured by possessing a system $B$ such that $I(A, B)=k$, implies that one can gain up to $k$ extra bits about actions on $A$. Thus for the one-time pad, a shared mutual information of 1 allows Alice to securely communicate a single bit to Bob. Meanwhile in quantum dense coding, Alice and Bob initially share a Bell state - such that $I(A, B)=2$. Thus, Bob can harness his memory to gain 2 exclusive bits about Alice's actions on $A$.

\section{A. The Role of Discord}

Recall that we can separate correlations into two components, i.e., we can write $I(A, B)=J(A \mid B)+\delta(A \mid B)$, where $J(A \mid B)$ and $\delta(A \mid B)$ respectively represent the purely-classical correlations and the quantum correlations (discord). This fits well with the channel guessing game. The approach is to relate quantum and classical correlations with the quantum and classicality of Bob's memory. Specifically let us consider the three scenarios:

1. Memoryless Bob: Bob's memory is completely faulty. That is, Bob cannot access $B$ at all. Bob's resulting performance is then given by $I_{0}$ (as defined above).

2. Classical Bob: Bob's memory is classical. That is, Bob is required to measure any $\rho_{b}$ given to him with respect to some orthogonal basis, and stored the measurement results in place of $\rho_{b}$. Denote Bob's resulting performance by $I_{c}$.

3. Quantum Bob: Bob has unrestricted quantum information processing, and can (i) store $\rho_{b}$ without error, and (ii) coherently interact his memory with the system of interest. Bob's resulting performance is given by $I_{q}$.

Cases 1 and 3 have been outlined above. Our focus here is thus case 2. The rationale is that a classical memory should be able to make use of purely classical correlations, but not quantum correlations. Therefore, we would expect discord to be related with the performance gap, $I_{q}-I_{c}$, between quantum and classical Bob. This problem was studied in $\mathrm{Gu}$. et. al. 2], where they established that

$$
J(A \mid B)-\tilde{J}(A \mid B) \leq I_{c}-I_{0} \leq J(A \mid B) .
$$

Here $\tilde{J}(A \mid B)$ and $\tilde{J}(A \mid B)$ represent the classical correlations in $\rho_{A B}$ and $\tilde{\rho}_{A B}$. The equation describes an interesting connection between discord and the performance advantage of having quantum-over-classical memory. That is, introducing $\delta(A \mid B)-\tilde{\delta}(A \mid B)$ as the discord difference before and after encoding, we get

$$
\Delta \delta(A \mid B)-\tilde{I}(A, B) \leq I_{q}-I_{c} \leq \Delta \delta(A \mid B),
$$

where $\tilde{I}(A \mid B)$ is the mutual information of $\tilde{\rho}$. Consider now any encoding that attempts to communicate the maximum amount of information (known as a maximal encoding). In this scenario, $\tilde{\rho}_{A}$ is maximally mixed, $\tilde{\delta}=\tilde{I}=0$, and thus we have:

1. $I_{0}=1-S(A)$ : a memoryless Bob can only access the local memory available on $A$. That is, the maximum amount of information Bob can learn about what happens to $A$ is exactly the negentropy of $A$.

2. $I_{c}=I_{0}+J(A \mid B)$ : a classical Bob can learn an additional $\Delta_{c}=J(A \mid B)$ bits of information about actions on $A$. That is, he can exactly take advantage of the classical correlations between $A$ and $B$.

3. $I_{q}=I_{0}+I(A, B)$ : a quantum Bob can take advantage of the full correlations between $A$ and $B$. As such, his performance advantage over the classical case is exactly $\delta(A \mid B)$, the discord between $B$ and A.

These relations capture an operational interpretation of discord $\delta(A \mid B)$ as how much purely quantum mechanical knowledge $B$ has about $A$. An example if given in Fig. 1

\section{B. Example: Certifying Entangling Gates without Entanglement}

The interpretation of discord as quantum knowledge can be applied to verify whether someone is in possession of entangling gates, as also experimentally realized by using polarization photons [3. Consider the case where 


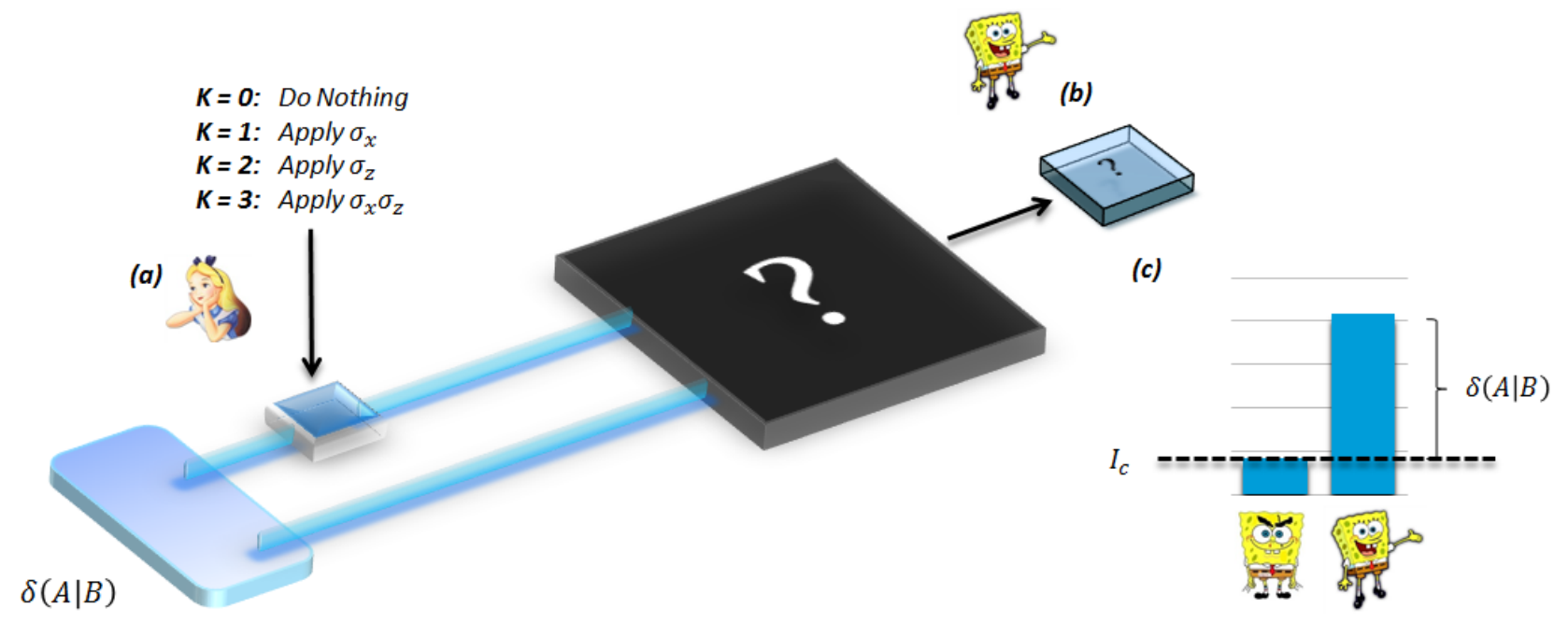

Figure 1. Example on Two Qubits. Consider the special case where Alice and Bob share a correlated state on two qubits, $A$ and $B$, with discord $\delta(A \mid B)$. Alice then encodes a random variable $K$ governed by a uniform distribution over $\{0,1,2,3\}$ by applying one of four possible unitaries, $I, \sigma_{x}, \sigma_{z}$ or $\sigma_{x} \sigma_{z}$ and challenges Bob to estimate $K$. In this scenario, the encoding is maximal, and Bob's performance gain when using quantum in the place of classical memory is given exactly by $\delta(A \mid B)$. This protocol has been experimentally implemented by by Almeida et.al. [3].

Bob claims that he is capable of building entangling twoqubit gates. How can Alice verify that Bob is telling the truth - without being able to generate entanglement herself?

The inability for classical processors to harness quantum knowledge suggests an immediate solution. Suppose now Alice prepares some discord, two-qubit state, $\rho_{A B}$. She can then perform the protocol above, using a specific encoding scheme that encodes two bits, $a, b \in\{0,1\}$, onto $A$, by applying the unitary $U=X^{a} Z^{b}$, where $X$ and $Z$ are standard Pauli operators. This corresponds to a scenario where $\tilde{\rho}_{A}=I / 2$ is maximally mixed. Alice then challenges Bob guess $a$ and $b$. Bob's performance is then characterized by the mutual information between the encoded bits, and that of Bob's guess.

In the 2 qubit case, it can be shown that if Bob is incapable of synthesizing entangling two-qubit gates, then he cannot exceed the performance level of $I_{c}$. As such any performance exceeding $I_{c}$ implies that Bob is capable of some entangling operations. Thus, discord can be used as a way of certifying entanglement without entangling gates.

\section{DISCORD IN QUANTUM KEY DISTRIBUTION}

Quantum discord also plays an important role in private communications and quantum key distribution (QKD) [4 6]. The fact that it must be non-zero is intuitive: Quantum discord and its geometric formulation are connected with the concept of non-orthogonality, which is the essential ingredient for QKD. A scenario where this is particularly evident is device-dependent (or trusteddevice) QKD. This includes all those realistic situations where the noise affecting the local devices is assumed to be trusted. For instance this can be detection noise (genuine inefficiency or noise added by the parties $[7,8]$ ) or preparation noise, as in the settings of untrusted-relay QKD [9, 10] and thermal-QKD [11 15]. Such trusted noise may be so high to prevent any entanglement distribution, but still a secure key can be extracted due to non-zero discord.

Any QKD protocol can be recast into a measurementbased scheme, where Alice sends Bob part of a bipartite state, then subject to local detections. Let us describe a device-dependent protocol in this representation. In her private space, Alice prepares two systems, $A$ and $a$, in a generally mixed state $\rho_{A a}$. This state is purified into a 3-partite state $\Phi_{P A a}$ with the ancillary system $P$ being inaccessible to Alice, Bob or Eve. This system accounts for the trusted noise in Alice's side. Then, system $b$ is sent to Bob, who gets the output $B$ after the channel (eavesdropping). Bob's output $B$ is assumed to be affected by other local trusted noise in Bob's private space (denoted as $P$ as before). Finally, from the shared state $\rho_{A B}$, Alice and Bob extract two correlated variables by applying suitable measurements. On the output data, they perform error correction and privacy amplification with the help of one-way CC, which can be either forward (direct reconciliation, $>$ ), or backward (reverse reconciliation, ४).

They finally extract a key at a rate $R=\max \left\{R_{\boldsymbol{}}, R_{\mathbf{4}}\right\}$, maximised between the reconciliations. Now we have [16]

$$
E_{D}(A, B) \leq R \leq E_{D}(A, B)+I(A B, P)
$$


where $E_{D}(A, B)$ is the one-way distillable entanglement for systems $A$ and $B$, as quantified by the maximum between the coherent [17, 18 and reverse coherent information [19, 20, while $I(A B, P)$ is the quantum mutual information between $A B$ and the trusted-noise system $P$. From Eq. (7), we see that the existence of $P$ is necessary in order to have $R>0$ in the absence entanglement (i.e., for $E_{D}=0$ ). Indeed it is easy to find discord-based Gaussian QKD protocols for which this is possible [16. According to Eq. (7), the absence of $P$ implies $R=E_{D}$, so that secure key distribution becomes equivalent to entanglement distillation [21].

In the absence of trusted noise, we have ideal QKD protocols where all the noise in the global output state is partly controlled by the parties and partly by Eve. In this setting, quantum discord becomes a simple upper bound for the key rate. In fact, for any ideal QKD protocol in direct or reverse reconciliation, we may write [16]

$$
R \leq \max \{\delta(A \mid B), \delta(B \mid A)\},
$$

where $\delta(A \mid B)$ and $\delta(B \mid A)$ are the two types of discord. Surprisingly, for the important practical case of a lossy channel [6] with transmissivity $\eta$, such as an optical fiber or a free-space link, the previous bound becomes tight. This is due to a combination of elements. First of all, we may always write 16

$$
R_{\triangleleft}=\delta(B \mid A)-E_{F}(B, E),
$$

where $E_{F}(B, E)$ is the entanglement of formation between Bob and Eve. Second, the Stinespring dilation of a lossy channel is a beam splitter with transmissivity $\eta$, mixing the Alice's input state with a vacuum environmental mode. For this reason, Bob and Eve's output state is not entangled, i.e., $E_{F}(B, E)=0$. Therefore, in a lossy channel, we always have

$$
R_{\triangleleft}=\delta(B \mid A) .
$$

Most importantly, one can prove [22] that the maximum discord $\delta_{\max }(B \mid A)$ that can be distributed to the parties through the lossy channel coincides with the secret-key capacity $K$ of the lossy channel (where this capacity is generally defined assuming the most general feedback-assisted protocols for key generation, based on unlimited two-way CC and adaptive local operations). In fact, Ref. 22] showed that

$$
K(\eta)=\delta_{\max }(B \mid A)=-\log _{2}(1-\eta),
$$

which provides the ultimate rate-loss scaling for bosonic secure communications, approximately $1.44 \eta$ secret bits per channel use for high loss (i.e., at long distances)

The proof Eq. (11) is based on several ingredients. First of all, it exploits the technique of teleportation stretching, devised in Ref. 22 for point-to-point quantum/private communications, and then extended in Ref. 23] to quantum repeaters and communication networks, and in Ref. 24 to quantum metrology and channel discrimination. In this technique, an arbitrary adaptive protocol for quantum/private communication is simplified into a much simpler non-adaptive form, providing the same output state as the original one. The advantage is that such output state is now decomposed in the form $\bar{\Lambda}\left(\rho_{\mathcal{E}}^{\otimes n}\right)$, where $\bar{\Lambda}$ is a trace-preserving LOCC, $\rho_{\mathcal{E}}$ is the Choi matrix [25] of the channel $\mathcal{E}$ (to be defined as suitable limit for a lossy channel), and $n$ is the number of uses of the channel. This decomposition is possible because the lossy channel is covariant with respect to the displacement operators and therefore can be simulated by means of continuous variable quantum teleportation [26, 27. In other words, the lossy channel is a specific example of teleportation-covariant channel [22].

The second ingredient is introduction of the channel's relative entropy of entanglement $E_{R}(\mathcal{E})$, which extends the original definition for quantum states 28 30 to quantum channels. Ref. 22 proved that, for any channel $\mathcal{E}$, the secret-key capacity satisfies the bound $K(\mathcal{E}) \leq E_{R}(\mathcal{E})$ (see also Ref. [31]). For the specific case of the lossy channel, one may combine the Choi-decomposition of the output $\bar{\Lambda}\left(\rho_{\mathcal{E}}^{\otimes n}\right)$ together with the properties of the relative entropy of entanglement to prove that $K(\eta) \leq E_{R}\left(\rho_{\mathcal{E}}\right)$. The latter term is the relative entropy of entanglement of the asymptotic Choi matrix of the lossy channel and must be computed as a limit over a sequence of two-mode squeezed vacuum states [22. This procedure leads to the upper bound

$$
K(\eta) \leq-\log _{2}(1-\eta) .
$$

Since the upper bound is achievable by a suitable Gaussian protocol in reverse reconciliation [16, 19, we then achieve Eq. (11). The proof can be easily extended to include the two-way quantum capacity, so that we also have $K(\eta)=Q_{2}(\eta)[22$.

\section{CONCLUSIONS}

In this brief review, we have discussed the role that quantum discord plays in two interesting settings. First of all, we considered the scenario of a bipartite system consisting of a system of interest, $A$, and a memory system $B$, such that their correlations, $I(A, B)$, represent knowledge $B$ has about $A$. This knowledge can be harnessed by a person in possession of $B$ to gain extra information about what performed on $A$. In this context, we outlined how discord is captured in the quantum component of such knowledge - measuring the component of $I(A, B)$ that is useful only when $B$ can be stored in quantum memory.

We then reviewed how quantum discord can be seen as a primitive for quantum cryptography, where it plays a double role. It is the bipartite resource which is exploited in trusted-noise QKD, where the presence of such noise may prevent the exploitation of quantum entanglement but not the distribution of a secret key. Then, quantum discord provides a general upper bound to the key rate in the ideal case when trusted noise is absent. In particular, this bound is achievable in the important case of 
lossy bosonic communications. In this setting, the maximum discord that two remote parties can generate at the two ends of a lossy channel corresponds exactly to the maximum number of secret bits that they can generate through the channel by means of the most general adaptive protocols for QKD.
[1] M. Berta et al., Nature Physics 6, 659-662.(2011).

[2] M. Gu et al., Nature Physics 8, 671-675 (2012).

[3] M. Almeida et al., Phys. Rev. A 89, 042323 (2014).

[4] N. Gisin, G. Ribordy, W. Tittel, and H. Zbinden, Rev. Mod. Phys. 74, 145 (2002).

[5] V. Scarani et al., Rev. Mod. Phys. 81, 1301, (2009).

[6] C. Weedbrook, S. Pirandola, R. Garcia-Patron, N. J. Cerf, T. C. Ralph, J. H. Shapiro, and S. Lloyd, Rev. Mod. Phys. 84, 621 (2012).

[7] R. Renner, N. Gisin, and B. Kraus, Phys. Rev. A 72, 012332 (2005).

[8] S. Pirandola, R. García-Patrón, S. L. Braunstein, and S. Lloyd, Phys. Rev. Lett. 102, 050503 (2009).

[9] S. L. Braunstein and S. Pirandola, Phys. Rev. Lett. 108, 130502 (2012).

[10] S. Pirandola, C. Ottaviani, G. Spedalieri, C. Weedbrook, S. L. Braunstein, S. Lloyd, T. Gehring, C. S. Jacobsen, and U. L. Andersen, Nature Photonics 9, 397-402 (2015).

[11] R. Filip, Phys. Rev. A 77, 022310 (2008).

[12] V.C. Usenko and R. Filip, Phys. Rev. A 81, 022318 (2010).

[13] C. Weedbrook, S. Pirandola, S. Lloyd, and T.C. Ralph, Phys. Rev. Lett. 105, 110501 (2010).

[14] C. Weedbrook, S. Pirandola, and T.C. Ralph, Phys. Rev. A 86, 022318 (2012).

[15] C. Weedbrook, C. Ottaviani, and S. Pirandola, Phys. Rev. A 89, 012309 (2014).

[16] S. Pirandola, Sci. Rep. 4, 6956 (2014).
[17] B. Schumacher and M.A. Nielsen, Phys. Rev. A 54, 2629 (1996).

[18] S. Lloyd, Phys. Rev. A 55, 1613 (1997).

[19] S. Pirandola, R. García-Patrón, S.L. Braunstein, and S. Lloyd, Phys. Rev. Lett. 102, 050503 (2009).

[20] R. García-Patrón, S. Pirandola, S. Lloyd, J.H. Shapiro, Phys. Rev. Lett. 102, 210501 (2009).

[21] A.K. Ekert, Phys. Rev. Lett. 67, 661 (1991).

[22] S. Pirandola, R. Laurenza, C. Ottaviani, and L. Banchi, Nat. Commun. 8, 15043 (2017); See also arXiv:1510.08863 (2015) and arXiv:1512.04945 (2015).

[23] S. Pirandola, "Capacities of repeater-assisted quantum communications", Preprint arXiv:1601.00966 (2016).

[24] S. Pirandola, and C. Lupo, Phys. Rev. Lett. 118, 100502 (2017).

[25] C. Choi, Linear Algebra Appl. 10, 285-290 (1975).

[26] S. L. Braunstein, and H. J. Kimble, Phys. Rev. Lett. 80, 869-872 (1998).

[27] S. Pirandola et al., Nature Photon. 9, 641-652 (2015).

[28] V. Vedral, M. B. Plenio, M. A. Rippin, and P. L. Knight, Phys. Rev. Lett. 78, 2275-2279 (1997).

[29] V. Vedral, and M. B. Plenio, Phys. Rev. A 57, 1619 (1998).

[30] V. Vedral, Rev. Mod. Phys. 74, 197 (2002).

[31] R. Laurenza, S. L. Braunstein, and S. Pirandola, "Finiteresource teleportation stretching for continuous-variable systems", arXiv:1706.06065 (2017). 